\title{
Magnetic resonance diffusion tensor imaging of acute spinal cord injury in spinal trauma
}

\author{
Radwa Mohamed Diaa Eldeen Abd Alsamee Alkadeem', Mohammad Hassan Rashad El-Shafey², \\ Ali Ebrahim Mohammad Seif Eldein ${ }^{2}$ and Hanan Ahmad Nagy ${ }^{2^{*}}$
}

\begin{abstract}
Background: It was important to develop a non-invasive imaging technique for early evaluation of spinal cord integrity after injury; MRI was the method of choice for evaluation of any cord abnormalities. However, some patients have symptoms with no detectable abnormalities by MRI. The purpose of our study was to assess the role of diffusion tensor MRI in evaluating the integrity of spinal cord fibers in case of spinal trauma.

Results: Out of the studied 30 patients, conventional MRI revealed abnormalities in the spinal cord in 23 patients (76.67\%), diffusion tensor tractography revealed abnormalities in the spinal cord in 27 patients (90\%), the mean FA value at the level of injury $(0.326 \pm 0.135)$ was less than the mean FA value $(0.532 \pm 0.074)$ in control group ( $p$ value $<0.001)$, and the mean ADC value at the level of injury (1.319 \pm 0.378$)$ was less than the mean ADC value $(1.734 \pm$ 0.768) in the control group. FA was sensitive than ADC in the detection of the spinal cord abnormalities with a sensitivity of $93.33 \%$ versus $67.66 \%$ respectively.

Conclusion: DTI can be used to detect structural changes of spinal cord white matter fibers in acute spinal cord injury. A significant decrease of fractional anisotropy and apparent diffusion coefficient has been found at the site of spinal cord injury.
\end{abstract}

Keywords: Diffusion tensor imaging (DTI), Fractional anisotropy (FA), Spinal cord injury

\section{Background}

Traumatic spinal cord injury (TSCI) is one of the most distressing injuries in human beings. It is associated with high mortality and disability, and long-term costly rehabilitation treatment. The degree of eventual neurological damage and deficit depends on the level, severity, and extent of injury that may have a considerable negative influence on the life rate and efficiency of these patients [1].

In the emergency setting, it is mandatory for the patients to have instant imaging after elementary resuscitation. MRI is confined for those patients with posttraumatic myelopathy (spinal cord dysfunction) or in patients having symptoms that cannot be explained by

\footnotetext{
* Correspondence: hanan.nagy84@hotmail.com

${ }^{2}$ Faculty of Medicine, Tanta University, Tanta, Egypt

Full list of author information is available at the end of the article
}

findings on radiographs or $\mathrm{CT}$, or with lack of a credible neurologic exam $[2,3]$.

Magnetic resonance imaging (MRI) has a pivotal role in evaluating spinal trauma as bone marrow, soft-tissue, and spinal cord abnormalities that may not be evident by other imaging modalities. Early detection facilitates a proper instant diagnosis, and urgent management [4].

Magnetic resonance imaging (MRI) has the upper hand in detecting neural and extraneural injuries; disc herniations, epidural hematomas, spinal cord edema, contusion, hemorrhage, and ischemia. This is attributed to its high-contrast resolution, multiplanar capabilities, and various pulse sequences that make it an ideal method for diagnosing spinal trauma [5].

However, conventional MRI is limited in giving optimum information about the integrity of spinal cord 
tissue itself due to the non-specificity of the signal intensity changes that are not corresponding directly with erratic physiological changes. This explains the poor correlation of conventional MRI findings with neurological and functional ailments in different spinal cord pathologies. So, it is important to use the newly developed MRI sequences for optimum assessment of the injured spine and spinal cord $[5,6]$.

Diffusion tensor imaging (DTI) is a magnetic resonance imaging (MRI) technique potentially able to evaluate the microscopic structural organization of white matter fibers. DTI depends mainly on diffusion-weighted imaging (DWI) that is used to evaluate the free diffusion of the water molecules (Brownian motion) in the extracellular space of the live tissue. Apparent diffusion coefficient (ADC) is used as a quantitative measurement of any diffusion disturbances (restriction or increase). Fractional anisotropy (FA) is considered a marker of white matter integrity expressing the preferred direction of diffusion and its decrease indicates damage and degradation of the white matter tracts [7-9].

Diffusion tensor tractography (DTT) is another application of DTI, which provides three -dimensional presentation of white matter's diffusion in the direction of fibers (axons) [8].

The aim of this study is to assess the role of MR diffusion tensor imaging (DTI) in the evaluation of spinal cord injury in the setting of spinal trauma.

\section{Methods}

\section{Study population}

This prospective study was conducted on 30 patients with spinal trauma having neurological symptoms. The age of the selected patients ranged from 15 to 64 years with a mean of 37.467 years. They were referred from the neurosurgery department to the MRI unit in the radio-diagnosis department over a period from January 2019 to June 2020. A control group of 10 subjects was also included. To avoid bias, the control group age and sex were matching with those of the patients.

Approval of Research Ethics Committee (REC) and informed consent were obtained from all participants in this study after explanation of the benefits and risks of the procedure. Privacy and confidentiality of all patients' data were guaranteed. All data provisions were monitored and used for scientific purposes only.

The included criteria were all symptomatic patients with spinal trauma at the acute stage having neurological deficits by clinical examination (numbness, limb motor, or sensory deficit). No age or gender predilection.

Exclusion criteria were patients with chronic spinal trauma, or patients with contraindications to MRI examination as patients with any metallic prosthesis or artificial pacemakers or claustrophobic patients or uncooperative patients with mental and behavioral disorders.

\section{All the included participants were subjected to magnetic resonance imaging}

All MRI scans were performed using a 1.5-T GE (General Electric) machine (closed magnet).

\section{Data collection}

- Full medical history, including personal history, mode, and timing of trauma; onset, course, and duration of current illness; past history of previous spinal trauma, spinal disease, or spinal operations.

- Review of all previous investigations or radiological examination.

\section{Clinical examination}

Performed for all the studied patients by a neurosurgeon at the Neurosurgery Department including general examination and neurological examination for focal neurological deficits (motor and sensory) using The American Spinal Injury Association (ASIA) impairment scale (modified Frankel classification) as illustrated in Table 1 [10].

\section{Patient preparation}

- The patients were instructed to remove all metallic objects such as hairpins, coins, ear rings, etc.

- The patients were reassured after explanation of the procedure. They were informed about the length of the examination and asked to remain motionless.

\section{Patient position}

All patients were examined in the neutral supine position using a standard body coil.

Table 1 The American Spinal Injury Association (ASIA) impairment scale (modified Frankel classification) ASIA grade description [10]

\begin{tabular}{ll}
\hline A & $\begin{array}{l}\text { Complete no sensory or motor function persists in the } \\
\text { sacral segments S4-S5 } \\
\text { Bncomplete sensory and not motor function remains } \\
\text { below the neurological level, including the sacral } \\
\text { segments S4-S5 } \\
\text { Incomplete motor function is pertained below the } \\
\text { neurological level. More than half of the key muscles } \\
\text { below the neurological level possess a muscle grade } \\
\text { less than } 3 \\
\text { Incomplete motor function is restored below the } \\
\text { neurological level, and at least half of the key muscles } \\
\text { below the neurological level have muscle grade greater } \\
\text { than or equal to } 3\end{array}$ \\
& Normal sensory and motor functions remain normal \\
\hline
\end{tabular}




\section{MRI protocol}

The following MRI pulse sequences were included:

Routine MRI sequences:

$>$ SagittalT2WFSE: TR/TE: 2245/101, FOV: $33 \times 33$,

4-mm thickness, NEX: 4, and matrix: $320 \times 224$.

$>$ Sagittal T1WFSE: TR/TE: 340-560/9-10, FOV: $33 \times$

33, $4 \mathrm{~mm}$ thickness, NEX: 4 , and matrix: $320 \times 224$.

$>$ Axial T1: TR/TE: 424-524/3-7, FOV: $33 \times 33,4-\mathrm{mm}$

thickness, NEX: 4 , and matrix: $320 \times 224$.

$>$ Axial T2: TR/TE: 2230/101-150, FOV: $33 \times 33$, 4-

mm thickness, NEX: 4, and matrix: $320 \times 224$.

Diffusion tensor imaging MRI (DTI MRI):

It was obtained using a single-shot echo planar imaging $(\mathrm{EPI})$ sequence $\left(b\right.$ value $\left.=1000 \mathrm{~s} / \mathrm{mm}^{2}\right)$ using (15-20) diffusion encoding directions with scan time of approximately $10 \mathrm{~min}$. The image was acquired in the sagittal plane with an image matrix of $128 \times 128$, slice thickness of $4 \mathrm{~mm}$ with no inter-slice gap, and a field of view (FOV) of $280 \times 280 \mathrm{~mm}$. To enhance the signal-to noise ratio and reduce the phase fluctuations, magnitude-constructed images were repeated (averages $=4$ ) and temporally averaged.

\section{Image analysis}

- All MR images of patients and control subjects were analyzed by two radiologists with 16 and 10 years of MRI experience, blinded to the clinical data and laboratory indicators, in a standard clinical Picture Archiving and Diagnostic System workstation, and final decisions reached by consensus are reported.
- Conventional MR images were analyzed for assessment of any cord changes as abnormal signal intensity, anatomic location of the cord injury, or presence of edema or hemorrhage.

- Analysis of DTI data was carried out using an offline separate work station; three ROIs were placed across the cord (opposite, above, and below the level of the trauma).

- Quantitative analysis was performed to calculate the fractional anisotropy (FA) and apparent diffusion coefficient (ADC) from the ADC and FA maps for each ROI.

- Fiber Tracking Method: Three-dimensional white matter fiber tract maps were created. The data obtained from standard sequences, ADC map, FA map, and tractography pathway patterns were compared in patients and control group.

\section{Statistical analysis}

- The collected data were coded, processed, and analyzed using the SPSS (Statistical Package for Social Sciences) version 20 for Windows ${ }^{\circ}$ (IBM SPSS Inc., Chicago, IL, USA).

- Data were tested for normal distribution using the Shapiro Walk test. Qualitative data were represented as frequencies and relative percentages. Chi-square test $\left(\chi^{2}\right)$ was used to calculate the difference between qualitative variables as indicated. Quantitative data were expressed as mean \pm SD (Standard deviation) and range. Student's $t$ test was used to compare between two

Table 2 Distribution of the studied cases according to demographic data $(n=40)$

\begin{tabular}{|c|c|c|c|c|c|c|c|}
\hline & & \multicolumn{2}{|c|}{ Patient } & \multicolumn{2}{|c|}{ Control } & \multirow[t]{2}{*}{$x^{2}$} & \multirow[t]{2}{*}{$p$ value } \\
\hline & & No. & $\%$ & No. & $\%$ & & \\
\hline \multirow[t]{2}{*}{ Sex } & Male & 19 & 63.33 & 5 & 50.00 & \multirow[t]{2}{*}{0.556} & \multirow[t]{2}{*}{0.456} \\
\hline & Female & 11 & 36.67 & 5 & 50.00 & & \\
\hline \multirow[t]{6}{*}{ Age group } & $<20$ years & 3 & 10.00 & 1 & 10.00 & \multirow[t]{5}{*}{1.746} & \multirow[t]{5}{*}{0.782} \\
\hline & 20 to $<30$ years & 9 & 30.00 & 5 & 50.00 & & \\
\hline & 31 to $<40$ years & 5 & 16.67 & 1 & 10.00 & & \\
\hline & 41 to $<50$ years & 7 & 23.33 & 1 & 10.00 & & \\
\hline & $>50$ Years & 6 & 20.00 & 2 & 20.00 & & \\
\hline & & & & & & $T$ & $P$-value \\
\hline \multirow[t]{2}{*}{ Age (Years) } & Range & \multicolumn{2}{|c|}{$15-64$} & \multicolumn{2}{|l|}{$18-66$} & 0.482 & 0.633 \\
\hline & Mean \pm SD & \multicolumn{2}{|c|}{$37.467 \pm 14.371$} & \multicolumn{2}{|c|}{$34.900 \pm 15.279$} & & \\
\hline
\end{tabular}

$p$ value: probability

$X^{2}$ : chi-square test

$T$ : Student's $t$ test 

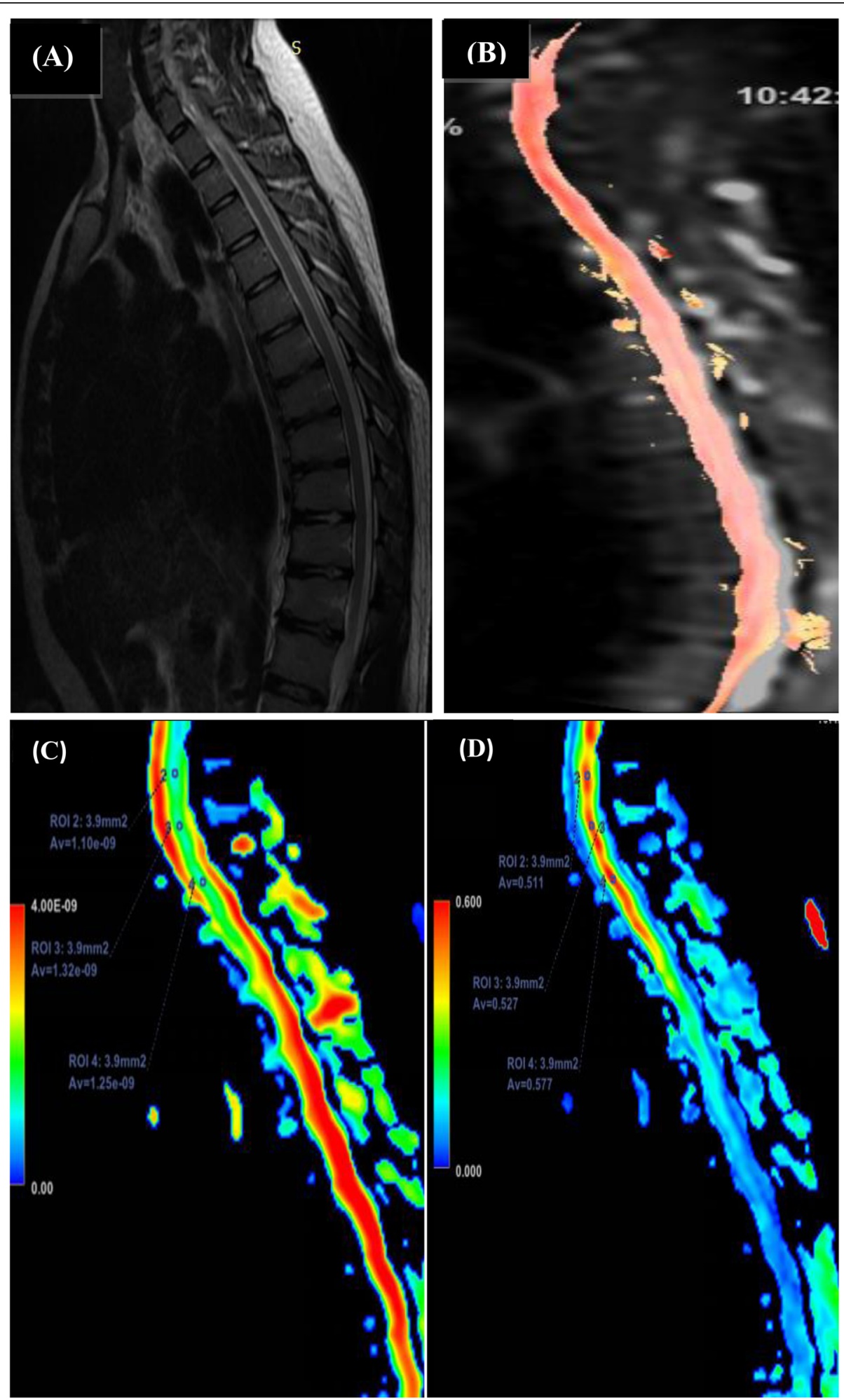

Fig. 1 a-d A 19 years old healthy male subject. a Sagittal T2-weighted MR image acquired at 1.5 T of a normal dorsal spine with normal osseous alignment, no abnormal marrow signal or abnormal cord signal. b Fiber tractography image shows intact white matter tract of dorsal spinal cord with no attenuation or interruption or displacement. c Sagittal color coded ADC map shows ADC values in different levels of the dorsal spine with no significant difference in between them (average 1.2). $\mathbf{d}$ Sagittal color coded FA map shows FA values in different levels of the dorsal spine with no significant difference in between them (average 0.538) 
independent groups of normally distributed variables (parametric data).

- Pearson's correlation $(r)$ was used to test the relationship between two quantitative, continuous variables.

- Spearman's correlation $(r)$ was used to test the correlation between two variables with nonparametric quantitative data.
- The receiver operator characteristic (ROC) curve was tested to calculate the diagnostic ability of quantitative variables in the prediction of categorical outcome.

- Probability ( $p$ value): $p$ value ${ }^{<} 0.05$ was considered significant, $p$ value ` 0.05 was considered insignificant, and $p$ value $<0.01$ was considered highly significant.
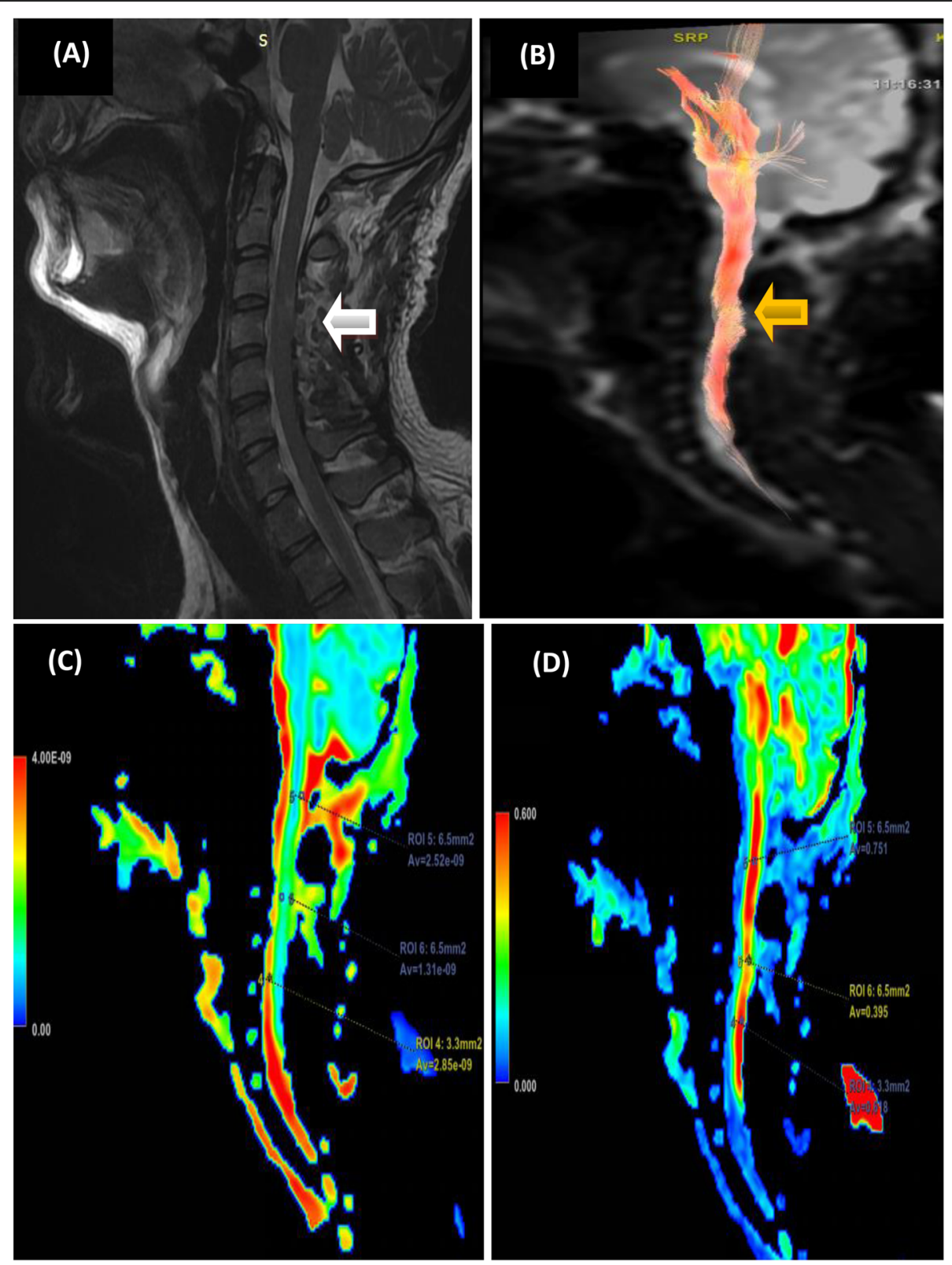

Fig. 2 a-d A 22-year-old male patient hit forcibly by a blunt object on his back. Neurologically, he was ASIA grade C. a Sagittal T2-weighted MR image acquired at $1.5 \mathrm{~T}$ shows abnormal spinal cord signal at the level of C4-5 (white arrow). b Fiber tractography image shows partially interrupted white matter tract of cervical spinal cord with attenuated fibers (yellow arrow). c Sagittal color coded ADC map image shows an ADC value at the injury level (1.31) lower than values above (2.52) and below (2.85) the injury level. $\mathbf{d}$ sagittal color coded FA map image shows lower FA value at the injury level (0.395) than values above (0.751) and below (0.678) the injury level 

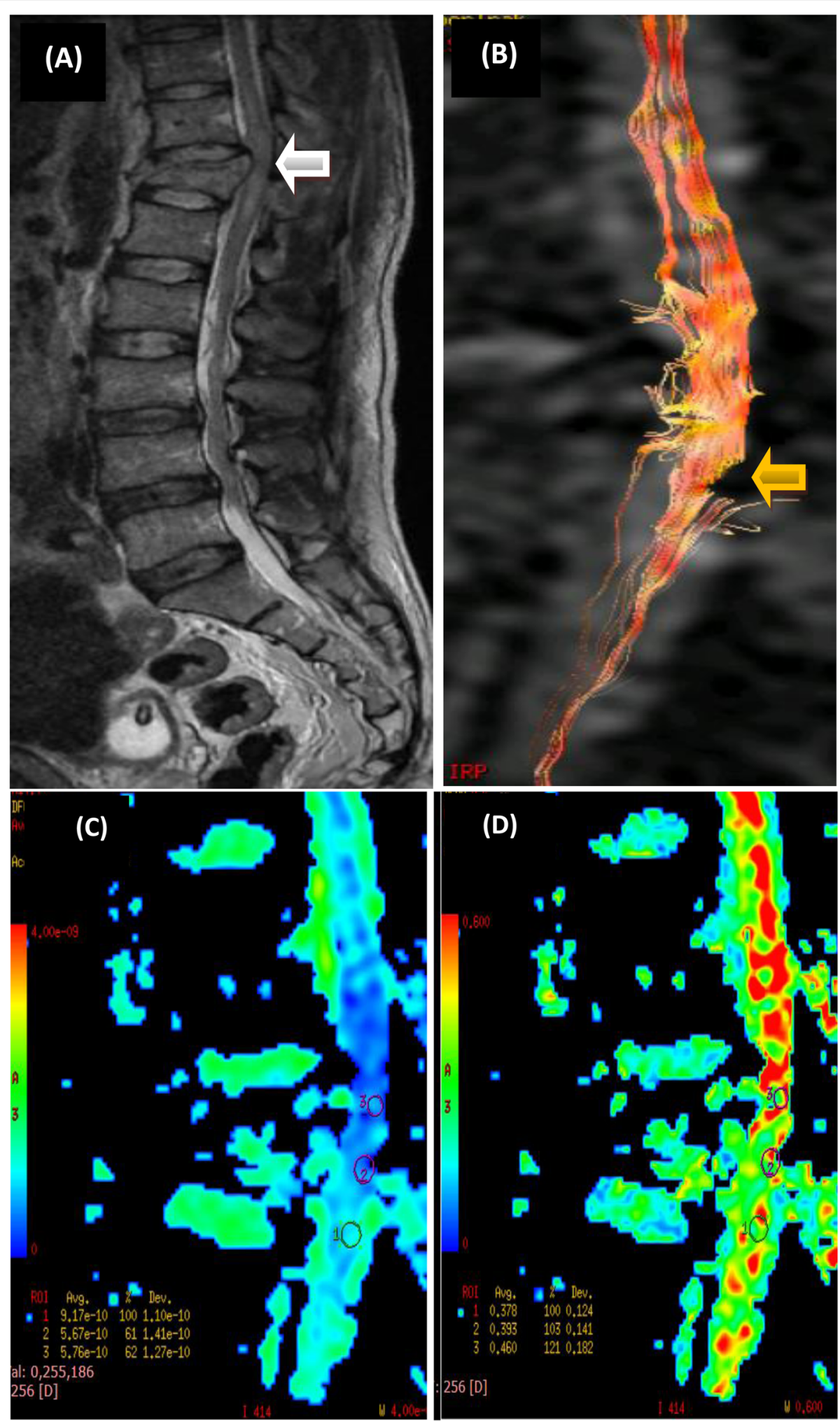

Fig. 3 a-d A 23-year-old male patient who fell from height. Neurologically, he was ASIA grade B. a Sagittal T2-weighted MR image acquired at 1.5 T shows wedged fracture at D12 vertebra with edema in the cord seen at the level of the injury (white arrow). $\mathbf{b}$ Fiber tractography shows incomplete interruption of the cord nerve fibers at the level of cord injury with apparently spared fibers (yellow arrow). c Sagittal color coded ADC map shows an ADC value at the injury level (1.41) higher than values above (1.27) and below (1.10) the injury level. $\mathbf{d}$ Sagittal color coded FA map shows lower FA values at and below the injury level (0.393) and (0.378) respectively than above the injury level (0.460) 


\section{Results}

This current prospective study included 2 groups: a control group of 10 individuals; 5 of them were males (50\%) and 5 were females $(50 \%)$ with their ages ranged from 18 to 66 years with a mean of $34.90 \pm 15.279$, and a patients group including 30 patients with spinal trauma and neurological deficits; 19 of them were males (63.3\%) and 11 were females (36.67\%) with their ages ranged from 15 to 64 years with a mean of $37.467 \pm 14.371$. The majority of patients were in the age group from 20 to 30 years; 9 patients representing 30\%. Demographic data of both studied control and patient groups is demonstrated in Table 2.
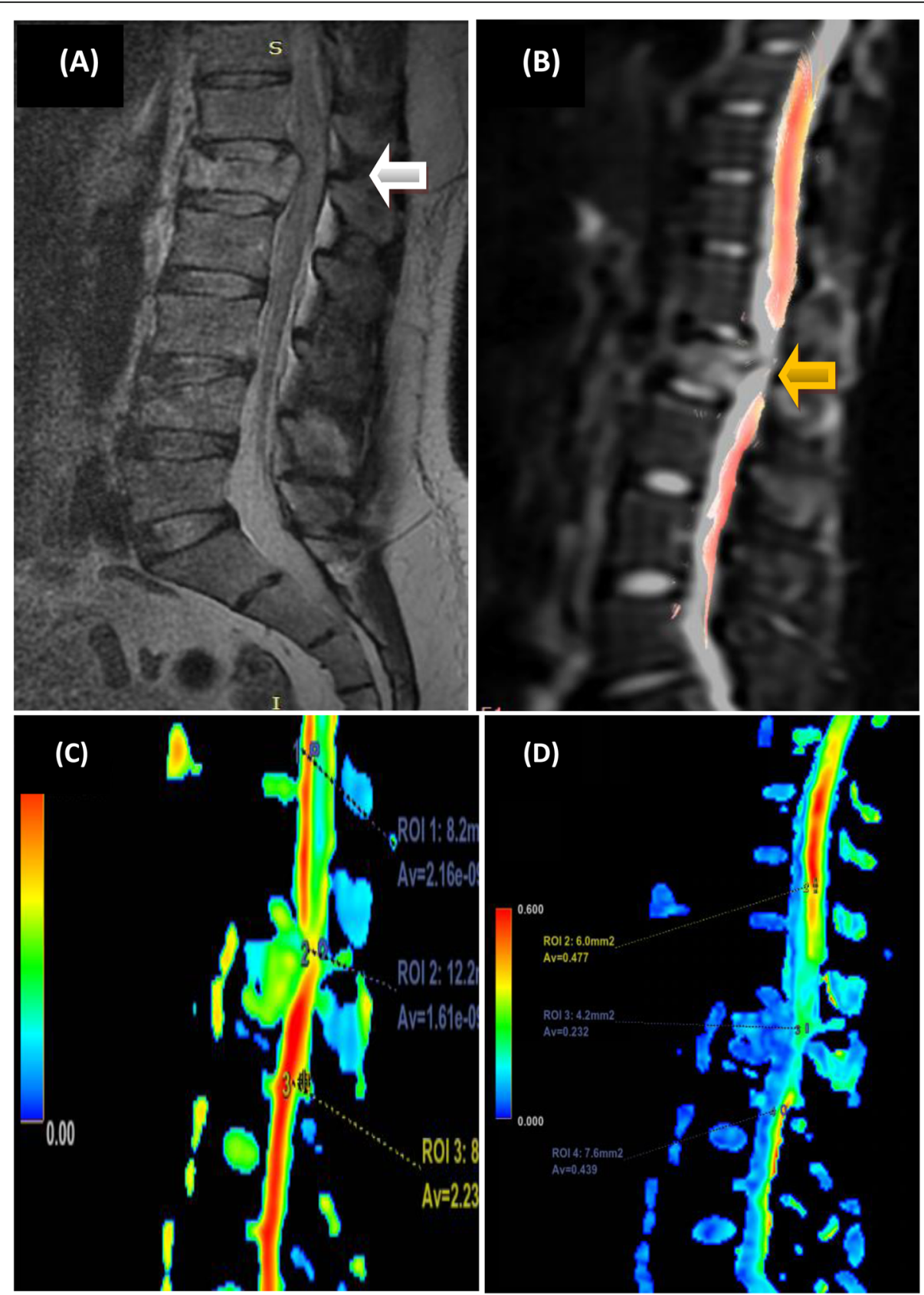

Fig. 4 a-d A 20-year-old female patient. Neurologically, she was ASIA grade B after a car accident. a Sagittal T2-weighted MR image acquired at 1.5 T shows wedged fracture of L1 vertebra compressing lower end of the spinal cord (white arrow). $\mathbf{b}$ Fiber tractography shows complete interruption in the spinal cord white matter tract (yellow arrow). c Sagittal color coded ADC map shows an ADC value at the injury level (1.61) lower than values above (2.16) and below (2.23) the injury level. $\mathbf{d}$ sagittal color coded FA map shows lower FA value at the injury level (0.232) than values above (0.477) and below (0.439) the injury level 
According to the etiology of spinal trauma, it was road traffic accident in 16 patients (53.33\%) (12 with motor vehicle collision and 4 with motorcycle collision), falling from height in 8 patient (26.67\%), sliding on the ground in 3 patients $(10 \%)$, hit by a blunt object in 2 patients (6.67\%) and gunshot in 1 patient (3.33\%).

After clinical examination according to the ASIA impairment scale, we found 6 patients (20\%) with grade A, 3 patients (10\%) with grade B, 8 patients $(26.66 \%)$ with grade $\mathrm{C}$, and 13 patients (43.33\%) with grade $\mathrm{D}$.
All examined patients $(n=30)$ were at the acute stage; the time interval between trauma and MRI examination was between 2 and $48 \mathrm{~h}$ with a mean of $10.333 \pm 13.817$.

After analysis of all MRI studies, 19 patients (63.33\%) had lumbar injuries, 7 patients (13.33\%) with dorsal injuries, and 4 patients $(23.33 \%)$ with cervical injuries. In the control group, the dorsal region was examined in 4 subjects, 3 subjects at the cervical region, and 3 subjects at the lumbar region $(p$ value $=0.177)$.
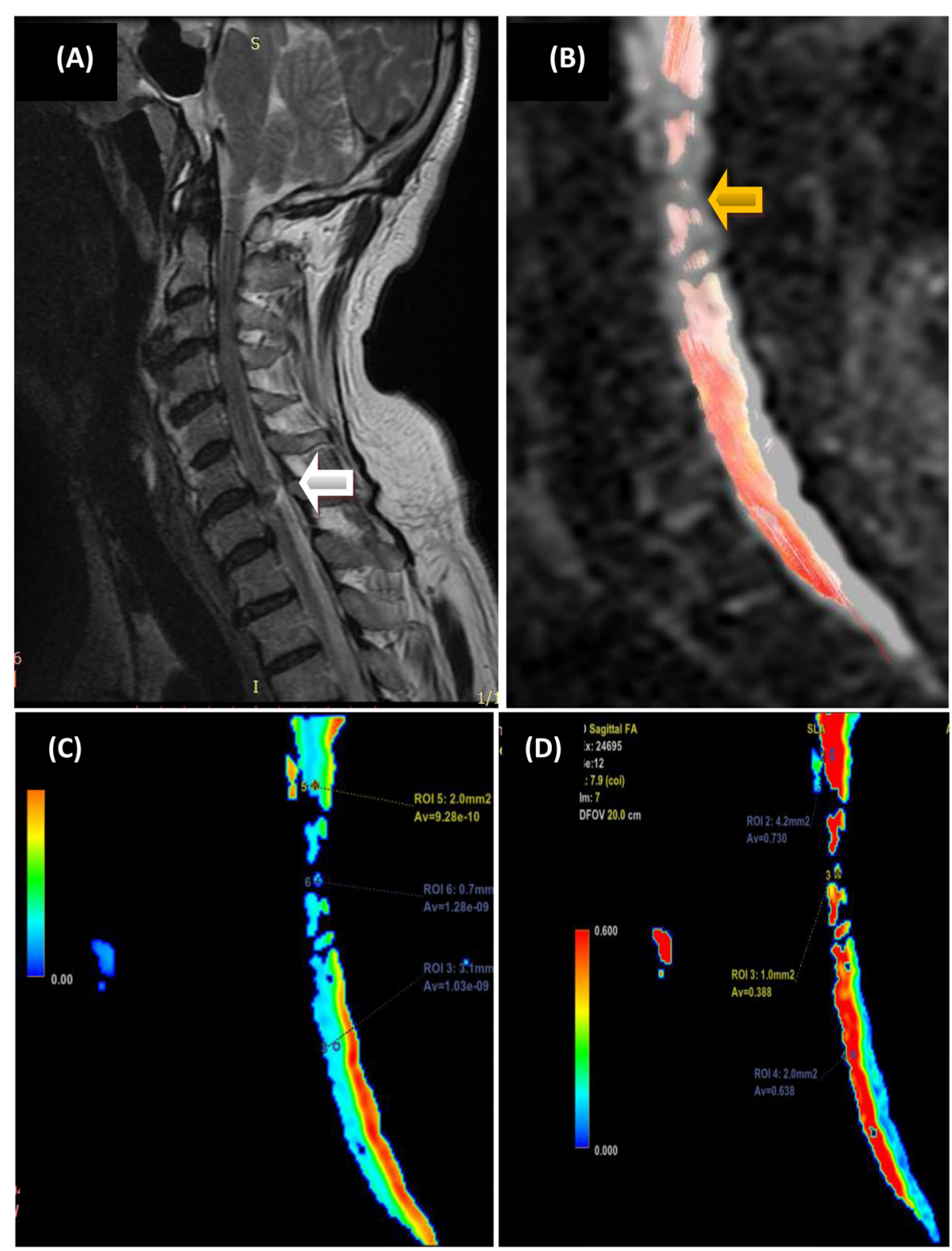

Fig. 5 a-d A 57 year old male patient. Neurologically, he was ASIA grade A after a car accident. a Sagittal T2-weighted MR image acquired at 1.5 T shows C6-7 traumatic disc herniation with abnormal spinal cord signal (white arrow). b Fiber tractography shows complete interruption of the cord nerve fibers at the level of cord injury(yellow arrow). c Sagittal color coded ADC map shows low ADC value at the injury level (1.28), above the injury level (0.928) and below the injury level (1.03). $\mathbf{d}$ Sagittal color coded FA map shows lower FA value at the injury level (0.388) than values above (0.730) and below (0.638) the injury level 
Conventional MRI revealed abnormalities in the spinal cord in 23 patients (76.67\%). In the remaining cases (23.33\%), the cord appeared normal, despite the presence of neurological deficit as determined by the ASIA impairment scale.

In conventional MRI, out of the 30 patients, 5 patients (16.6\%) had traumatic disc bulge, 24 patients $(80 \%)$ had secondary spinal canal stenosis, 23 patients $(76.6 \%)$ had cord compression, 23 patients $(76.6 \%)$ had cord signal changes, 24 patients $(80 \%)$ had reduced disc height, and 25 patients $(83.3 \%)$ had bone marrow edema.

Regarding DTI MR imaging, a qualitative tractographic analysis was performed in all patients and controls (DTT). It showed gross normal integrity of white matter tracts in the cord in all 10 controls (Fig. 1), while in the patient group, it revealed abnormalities in the spinal cord in 27 patients (90\%) (Figs. 2, 3, 4, and 5). In the remaining 3 patients $(10 \%)$, the cord appeared normal, despite the presence of neurological deficit as determined by the ASIA impairment scale.

Three patients (10\%) had gross normal appearance of the spinal cord in DTT image, 7 patients (23.33\%) had complete interruption, 7 patients (23.33\%) had just attenuation, 6 patients $(20 \%)$ had partial interruption, 4 patients $(10 \%)$ had displacement, and attenuation and 3 patients (10\%) had partial interruption and attenuation.

In the patient group, the mean FA value at the level of injury $(0.326 \pm 0.135)$ was less than the mean FA value $(0.532 \pm 0.074)$ in the control group, which was statistically significant $(p$ value $<0.001)$. However, no significant difference was found in the mean FA value above or below the level of injury in the patient group in comparison with the control group with $p$ values of 0.742 and 0.071 , respectively, as illustrated in Table 3 .

Table 3 Comparison of FA parameter between patients and control group

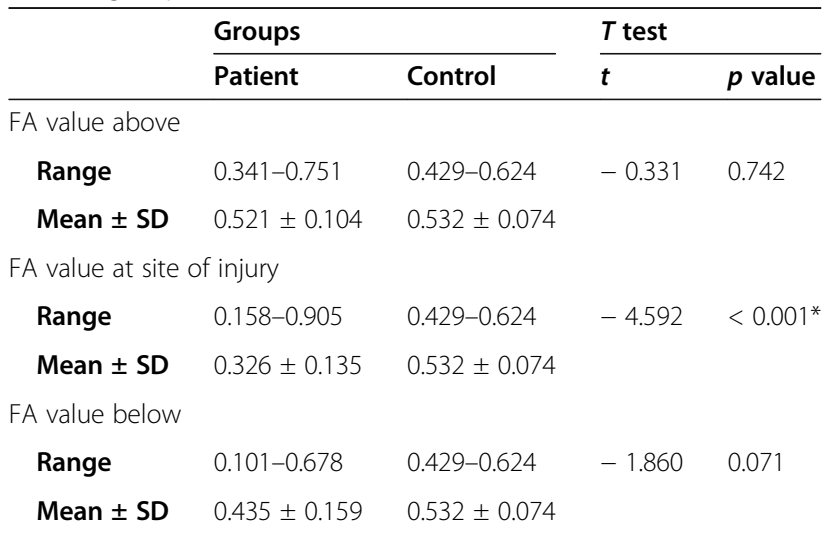

$T$ : Student's $t$ test

$p$ value: probability for independent samples $t$ test for association between patients and control group

*Significant $p$ value $(<0.05)$
The mean ADC value at the level of injury (1.319 \pm $0.378)$ was less than the mean ADC value $(1.734 \pm$ 0.768 ) in the control group, which was statistically significant ( $p$ value $0.028^{*}$ ). However, no significant difference was found in the mean ADC value above the level of injury or below the level of injury in the patient group in comparison with the control group with $p$ values of 0.563 and 0.589 , respectively, as shown in Table 4.

According to ROC analysis for detection of sensitivity and specificity of fractional anisotropy and apparent diffusion coefficient quantitative analysis of DTI MRI to detect the spinal cord abnormalities between patients and control group, we noticed that the ROC for FA gave AUC 0.957 while for ADC, it gave AUC 0.712 with sensitivity and specificity of FA $93.33 \%$ and $100 \%$ respectively versus sensitivity and specificity of ADC $66.67 \%$ and $80 \%$, respectively, as shown in Table 5 and Figs. 6 and 7.

Pearson's correlation test showed no significant correlation between age (years) and FA at the level of injury with a $p$ value of 0.436 . Also, no significant Pearson correlation was found between time since injury (hours) and FA at the level of injury with a $p$ value of 0.266 , as shown in Table 6.

Spearman's correlation test showed statistically significant ( $p$ value 0.006$)$ negative $(r$ value $=-0.487)$ correlation between FA values at the level of injury and clinical grading ASIA impairment scale (A, B, C, D, E) in order of decreasing sensorimotor deficit), as shown in Table 7 and Fig. 8.

\section{Discussion}

Diffusion tensor imaging (DTI) is a noninvasive MRI technique that measures the random motion of water molecules and provides information about the cellular integrity and pathology of anisotropic tissues. DTI can provide unique quantitative information on the microstructural features of white matter in the central nervous system [9].

This study was carried out on 30 patients presenting with spinal trauma and complaining from neurological symptoms and a control group of 10 subjects.

Fiber tractography (FT) is a valuable parameter of DTI measurement; it is a technique that uses specialized tracing algorithms to get a three-dimensional reconstruction of white matter tracts in the central nervous system. It is commonly used for evaluating fiber directions and defects in the brain and spinal cord. It can show the macroscopic orientation of fibers with dramatic representation of the disruption of tracts, which can be hardly seen on conventional MRI, allowing better delineation of damaged fiber tracts in the injured spinal cord $[11,12]$. 
Table 4 Comparison of ADC parameter between patients and control group

\begin{tabular}{|c|c|c|c|c|c|}
\hline \multirow[t]{2}{*}{$A D C$} & & \multicolumn{2}{|l|}{ Groups } & \multicolumn{2}{|l|}{$T$ test } \\
\hline & & Patient & Control & $\bar{T}$ & $\overline{p \text { value }}$ \\
\hline \multirow[t]{2}{*}{ ADC value above } & Range & $0.347-2.91$ & $0.87-3.65$ & 0.584 & 0.563 \\
\hline & Mean \pm SD & $1.875 \pm 0.623$ & $1.734 \pm 0.768$ & & \\
\hline \multirow[t]{2}{*}{ ADC value at site of injury } & Range & $0.567-2.75$ & $0.87-3.65$ & -2.278 & $0.028^{*}$ \\
\hline & Mean \pm SD & $1.319 \pm 0.378$ & $1.734 \pm 0.768$ & & \\
\hline \multirow[t]{2}{*}{ ADC value below } & Range & $0.576-3.62$ & $0.87-3.65$ & 0.544 & 0.589 \\
\hline & Mean \pm SD & $1.870 \pm 0.655$ & $1.734 \pm 0.768$ & & \\
\hline
\end{tabular}

T: Student's $t$ test

$p$ value: probability for independent samples $t$ test for association between patients and control group

*Significant $p$ value $(<0.05)$

In this study, diffusion tensor tractography (DTT) in the injury site was reconstructed in all patients and controls. Normal orientation of white matter tracts in the cord was visualized in all 10 controls in the form of one bundle of homogenous orange color suggestive of preservation of the integrity of white matter tracts. In the 30 patients with spinal trauma, routine MRI scanning could detect spinal cord signal changes in 23 cases (sensitivity $76.67 \%$ ), while on tractography, changes in cord integrity were observed in 27 cases in the form of distortion of homogenous orange color with or without interruption. In the remaining 3 patients, the spinal cord appeared grossly normal on qualitative tractographic analysis (Sensitivity 90\%).

D'souza, et al. [11] also found normal orientation of white matter tracts in the cord in all 30 controls. In 20 patients, only 10 patients routine had changes in cord signal intensity (Sensitivity 50\%), while tractography detected changes in cord integrity in 12 cases in the form of distortion of the homogenous color denoting disruption in cord integrity (Sensitivity 60\%). However, Wang, et al. [9] found that all patients with abnormalities in DTT had apparent cord abnormalities in cMRI.

In other studies, 3.0-T MR machines were used to measure the fiber density of selected fiber bundles depending on the number of fiber projections per voxel. The study of Wen, et al. [13] was conducted on 15 healthy adults and 7 myelopathic spinal cord patients with $b$ values of 0 and $600 \mathrm{~s} / \mathrm{mm}^{2}$, they measured the tract numbers from C1-7. They found a significant difference in track numbers between the healthy and myelopathic groups; $3064.40 \pm 482.38$ and $2282.71 \pm 293.80$ in healthy and myelopathic adults, respectively.

Fractional anisotropy (FA) is the value which signifies the anisotropic part of diffusion as it measures the tendency of water to spread in a preferred direction within a group of axons. It is a function of the axonal density and integrity of white matter fibers, as well as of their degree of myelination [14].

We found that FA values were the most sensitive parameter of DTI for assessment of TSCI (93.33\%). The mean FA values at injury level among patients $(0.326 \pm$ $0.135)$ were significantly lower than in controls $(0.532 \pm$ 0.074). This decrease could be attributed to anisotropic diffusion restriction in traumatized spinal cord. FA values indirectly measure the extent of myelination, so, higher FA values indicate the integrity of spinal nerves [11]. No significant difference was observed in the mean FA values above or below the injury level in our patients as compared with controls.

Hassen and El-Kholy [15] demonstrated a decrease in FA values in TSCI patients similar to our study. Regional measurements of FA values at 5 cord levels showed a significant decrease at the level of cord injury.

Czyz, et al. [16] also found that FA values in cases with TSCI were significantly lower as compared with the controls (0.48 and 0.55, respectively). Similarly, Rao, et al. [17] found decreased FA values in TSCI patients

Table 5 Analysis of the diagnostic ability of ADC and FA to differentiate between patients and control groups

\begin{tabular}{|c|c|c|c|c|c|c|c|c|}
\hline \multicolumn{9}{|c|}{ ROC curve between patient and control } \\
\hline \multicolumn{9}{|c|}{ Cutoff } \\
\hline \multicolumn{9}{|l|}{ FA value at site of injury } \\
\hline$A D C$ value at site of injury & $\leq 1.31$ & $66.67 \%$ & $80.00 \%$ & $90.9 \%$ & $44.4 \%$ & 0.712 & $54.7-84.4$ & $0.051^{*}$ \\
\hline
\end{tabular}

Sens. sensitivity, spec. specificity, PPV positive predictive value, NPV negative predictive value, AUC area under the curve, $C I$ confidence intervals $p$ value: probability for independent samples $t$ test for association between patients and control group

*Significant $p$ value $(<0.05)$ 


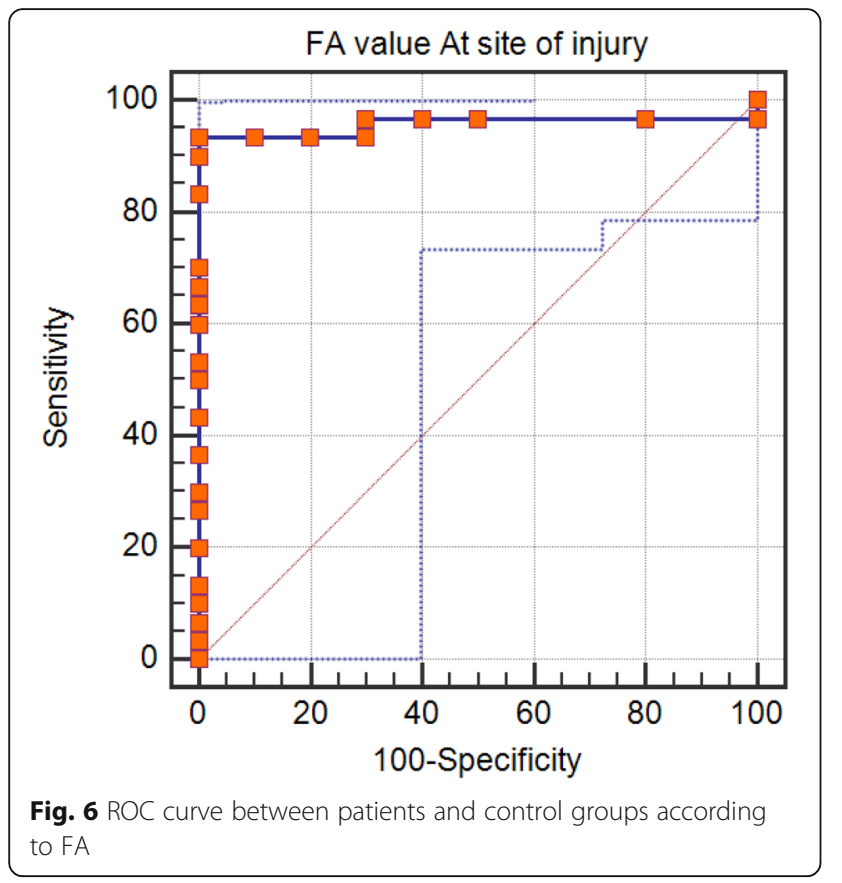

compared with neurologically intact volunteers $(0.220 \pm$ 0.121 and 0.545 to 0.601 respectively).

Our study was also in accordance with the findings of Shanmuganathan, et al. [18] and Cheran, et al. [19] who showed reduced FA values in patients with TSCI as compared with controls.

This study did not reveal any significant difference in mean FA values above or below the injury level. This matches with the same findings reported by D'souza, et al. [11]. However, Kamble, et al. [20] in their study

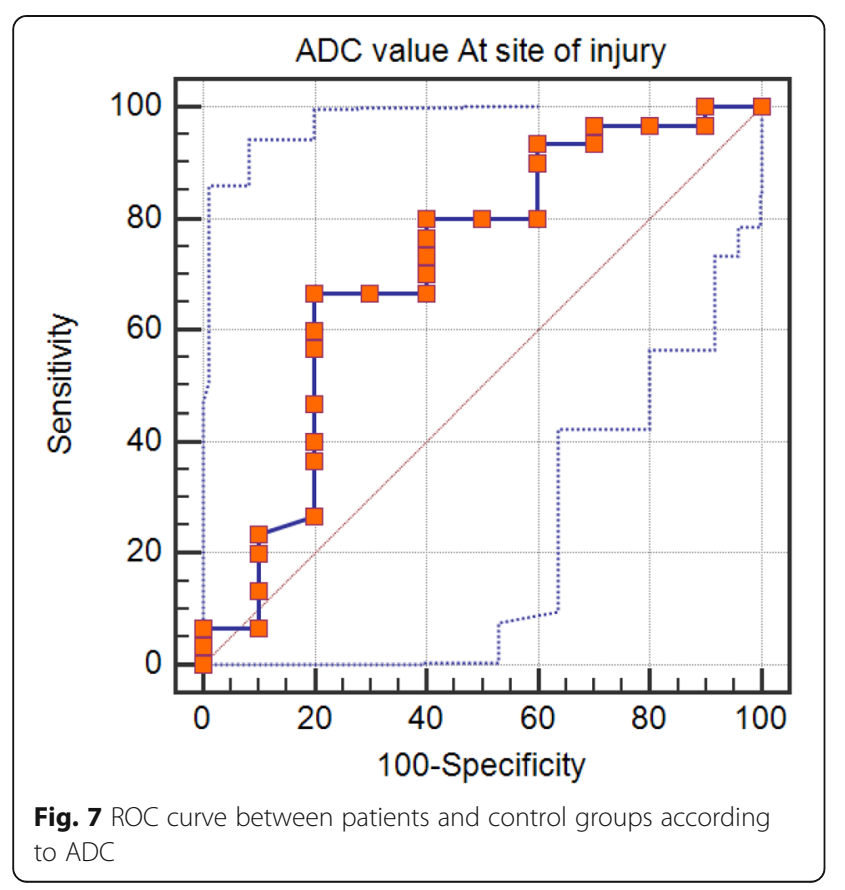

Table 6 Pearson correlation between age (years), time since injury (hours), and FA at the level of injury

\begin{tabular}{|c|c|c|c|c|}
\hline & \multicolumn{2}{|c|}{ Age (years) } & \multicolumn{2}{|c|}{ Time since injury (hours) } \\
\hline & $r$ & $p$ value & $R$ & $p$ value \\
\hline FA value at site of injury & -0.148 & 0.436 & -0.210 & 0.266 \\
\hline
\end{tabular}

showed that the FA values in the cord above and below injury level were considerably reduced. They concluded that, as a result of trauma, there was resultant descending as well as ascending Wallerian degeneration, and this fact leads to altered DTI metrics. Similar findings were also found by Mohamed, et al. [21] who conducted a study on DTI in children with spinal trauma.

The reason for the absence of any variation in DTI parameters above or below the level of injury in our study could probably be due to the timing of the imaging which was held soon after trauma in majority of our cases, whereas other studies have followed up the patients for a longer period of time sufficient for spread of axonal degeneration.

Using Spearman's correlation test between FA and ASIA impairment scale (A, B, C, D, E), we found that the lower the FA values, the worse the clinical condition $(r$ value $=-0.487)$. In the study of D'souza, et al. [11], they found a decrease in FA value reflecting axonal injury, and correlation with clinical status has been detected; the lower the FA values, the more severe is the injury with more serious neurological dysfunction and worse outcome.

The apparent diffusion coefficient (ADC) value was another parameter that we used in the current study; it refers to the overall diffusivity of the tissues whatever the number of barriers to water motion such as myelinated axons, cellular membranes, and extracellular molecules. It is extremely sensitive to the abnormalities typically seen in SCI; when combined with fiber tracking, the damaged areas of the spinal cord can be examined better than with T2-weighted imaging [22, 23].

We determined that the mean ADC value at the level of injury among cases (1.319 \pm 0.378$)$ was significantly lower as compared with values in controls (1.734 \pm 0.768). No significant difference was observed in mean $\mathrm{ADC}$ values above or below the injury level among the cases and controls.

Previous studies have also shown a decrease in ADC values in patients with acute TSCI in accordance with our study as Shanmuganathan, et al. [18] who reported that ADC is significantly decreased in patients with acute TSCI and patients with spinal cord hemorrhage exhibiting the greatest decrease and $\mathrm{Li} \mathrm{XH}$, et al. [24] who also found that the mean value of ADC was 
Table 7 Spearman's correlation between FA at the level of injury and ASIA impairment scale

\begin{tabular}{lll}
\hline & \multicolumn{2}{l}{ ASIA Impairment Scale } \\
\cline { 2 - 3 } $\boldsymbol{r}$ & -0.487 & $\boldsymbol{p}$ value \\
\hline FA value at site of injury & $0.006^{*}$ \\
\hline : Spearman's correlation & \\
patients and control group. & \\
*Significant $p$ value $(<0.05)$ &
\end{tabular}

significant decrease at $24 \mathrm{~h}$ and $72 \mathrm{~h}$ in the severely injured group $(p<0.05)$.

Ellingson, et al. [25] have reported lower ADC values in the cervical cord of patients with TSCI. They suggested that these low ADC values were due to the decrease in overall diffusion magnitude occurring away from the injury site resulting from the restructuring of the axons and widespread spinal cord degeneration mainly in chronic cases.

However, some other studies reported that ADC is non-significantly different at the site of injury and the control group as the study by Czyz, et al. [16] who found that the difference was not statistically significant $(p=0.28)$, but suggested that ADC may serve a potential prognostic factor.

In the present study, due to the significant difference in the FA and ADC values at the site of trauma among patients as compared with controls, it is logical to conclude that DTI with these 2 parameters is a valuable instrument in assessment of the spinal cord following TSCI. And after analyzing the diagnostic accuracy of both parameters, we realized that FA is more sensitive than $\mathrm{ADC}$ (93.33\% and $66.67 \%$ respectively) in diagnosing SCI and differentiating between patients and control groups.

Facon et al. [26] showed although ADC decreased in the majority of SCI patients, it was not as sensitive as FA in the detection of acute SCI. The authors Fiani et al. [27] and Song, et al. [28] suggested that the use of ADC should be restricted to chronic spinal cord compression. However, Shanmuganathan et al. [18] reported that ADC was the most sensitive marker of acute cervical cord injury and found it to be uniformly decreased in patients with cervical spine trauma.

Spinal cord DTI still has many limitations. The main limitation is its spatial resolution, which is affected by artifacts emerging from cardiac and respiratory motion and cerebrospinal fluid pulsation. Also, the technique is operator dependent especially FT that depends on a qualitative visual analysis by the radiologist. Furthermore, those patients with acute spinal trauma cannot bear up extra scan acquisition time in the MRI suite.

The main limitations in the current study are the small sample size and the difficulty of follow-up of the patients for assessment of axonal regeneration especially after treatment.

\section{Conclusion}

This study realized that DTI, with its quantitative indices, is a valuable tool in assessment of the cord injury in cases of spinal trauma when compared with conventional MRI. FA and ADC values were significantly decreased at the level of injury. FA is more sensitive and

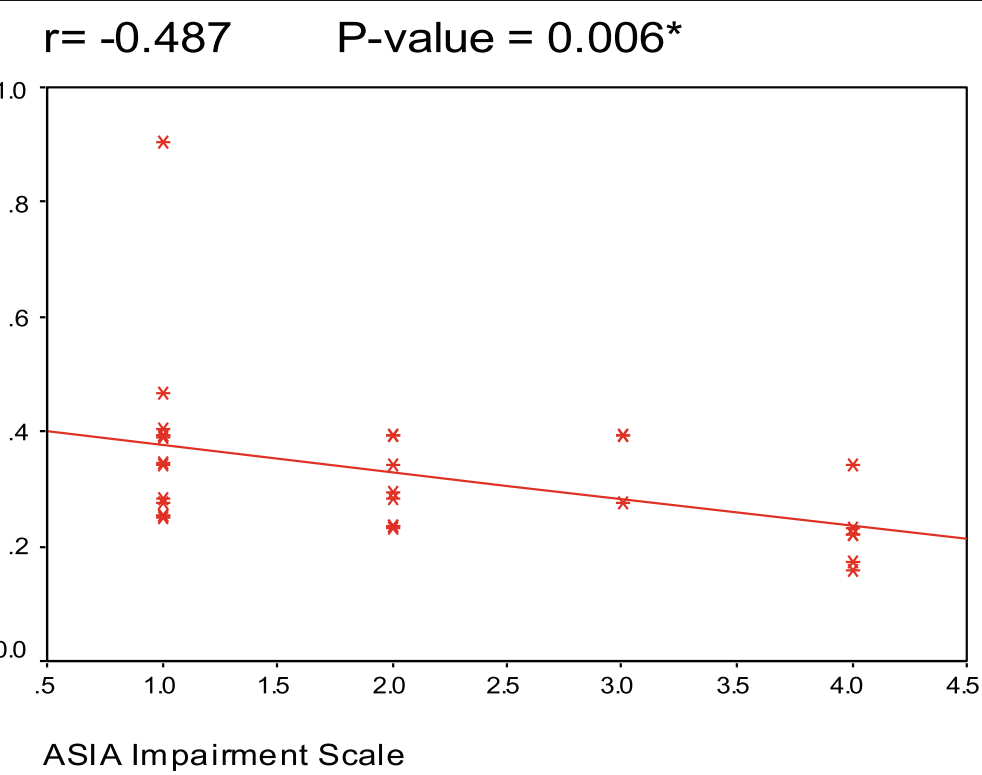

Fig. 8 Spearman's correlation between FA at the level of injury and ASIA impairment scale 
accurate in detecting abnormalities mainly at the site of injury.

We recommend using DTI as a routine investigation in spinal trauma complementary to cMRI for assessment of white matter integrity and prediction of functional outcome to reduce mortality and morbidity and use it in follow-up after treatment.

\section{Abbreviations}

ASIA: American Spinal Injury Association; ADC: Apparent diffusion coefficient; CMRI: Conventional magnetic resonance imaging; DTI: Diffusion tensor imaging; DTT: Diffusion tensor tractography; FA: Fractional anisotropy; SCl: Spinal cord injury; TSCl: Traumatic spinal cord injury

\section{Acknowledgements}

The authors thank all the participants for their cooperation and patience.

\section{Authors' contributions}

$\mathrm{MH}$ suggested the research idea, ensured the original figures and data in the work, minimized the obstacles to the team of work, correlated the study concept and design, and had the major role in analysis. RM collected data in all stages of the manuscript, performed data analysis. HA supervised the study with significant contribution to the design of the methodology, manuscript revision and preparation. AE correlated the clinical data of the patient and matched it with the findings, drafted and revised the work. All authors read and approved the final manuscript for submission.

\section{Funding}

No funding. Not applicable for this section.

\section{Availability of data and materials}

The authors confirm that all data supporting the finding of the study are available within the article and the raw data ad data supporting the findings were generated and available at the corresponding author on request.

\section{Declarations}

Ethics approval and consent to participate

Informed written consent taken from the patients and healthy volunteers; the study was approved by the ethical committee of Tanta University Hospital, Faculty of Medicine. Committee's reference number: 32740/11/18.

\section{Consent for publication}

All participants included in the research gave written consent to publish the data included in the study.

\section{Competing interests}

The authors declare that they have no competing interests.

\section{Author details}

${ }^{1}$ Health Insurance Hospital in Tanta, El-geish street, Tanta, Gharbya

Governorate, Egypt. ' Faculty of Medicine, Tanta University, Tanta, Egypt.

Received: 28 December 2020 Accepted: 22 February 2021

Published online: 02 March 2021

\section{References}

1. Hamid R, Averbeck MA, Chiang $\mathrm{H}$ et al (2018) Epidemiology and pathophysiology of neurogenic bladder after spinal cord injury. World J Urol 36(10):1517-1527

2. Copley PC, Jamjoom AAB, Khan S (2020) The management of traumatic spinal cord injuries in adults: a review. Orthop Trauma 34(5):255-265

3. Hauwe LVD, Sundgren PC, Flanders AE (2020) Spinal trauma and spinal cord injury (SCl). In: Juerg H, Huch K, Rahel A, et al. (eds.). Diseases of the Brain, Head and Neck, Spine 2020-2023: Diagnostic Imaging, (1st ed.) Springer, Cham, 19:231-240

4. Naik BR, Sakalecha AK, Savagave SG (2019) Evaluation of Traumatic Spine by Magnetic Resonance Imaging and Its Correlation with Cliniconeurological Outcome. J Emerg Trauma Shock 12(2):101-107
5. Magu S, Singh D, Yadav RK et al (2015) Evaluation of traumatic spine by magnetic resonance imaging and correlation with neurological recovery. Asian Spine J 9:748-756

6. Bozzo A, Marcoux J, Radhakrishna M et al (2011) The role of magnetic resonance imaging in the management of acute spinal cord injury. J Neurotrauma 28(8):1401-1411

7. Toktas ZO, Tanrıkulu B, Koban O et al (2016) Diffusion tensor imaging of cervical spinal cord: A quantitative diagnostic tool in cervical spondylotic myelopathy. J Craniovertebral Junction Spine 7(1):26-30

8. Sąsiadek MJ, Szewczyk P, Bladowska J (2012) Application of diffusion tensor imaging (DTI) in pathological changes of the spinal cord. Med Sci Monit 18(6):73-79

9. Wang W, Qin W, Hao N et al (2012) Diffusion tensor imaging in spinal cord compression. Acta Radiol 53(8):921-928

10. Tator CH, Rowed DW, Schwartz ML (1982) Sunnybrook cord injury scales for assessing neurological injury and neurological recovery in early management of acute spinal cord injury. In: Tator $\mathrm{CH}$ (ed) Early management of acute spinal cord injury. Raven Press, New York, pp 7-24

11. D'souza MM, Choudhary A, Poonia M et al (2017) Diffusion tensor MR imaging in spinal cord injury. Injury 48(4):880-884

12. Chang Y, Jung TD, Yoo DS et al (2010) Diffusion tensor imaging and fibre tractography of patients with cervical spinal cord injury. J Neurotrauma 27(11):2033-2040

13. Wen CY, Cui JL, Lee MP et al (2013) Quantitative analysis of fiber tractography in cervical spondylotic myelopathy. Spine J 13:697-705

14. Landi A, Innocenzi G, Grasso G et al (2016) Diagnostic potential of the diffusion tensor tractography with fractional anisotropy in the diagnosis and treatment of cervical spondylotic and posttraumatic myelopathy. Surg Neurol Int 7(25):705-707

15. Hassen RZ, El-Kholy SF (2018) Role of Diffusion Tensor Imaging (DTI) in Post Traumatic Spinal Cord Injury. Med J Cairo Univ 86(8):4429-4433

16. Czyz M, Tykocki T, Szewczyk P et al (2017) Application of diffusion tensor imaging in the prognosis of outcome after traumatic cervical spinal cord injury. J Spinal Stud Surg 1:25-28

17. Rao JS, Zhao C, Yang ZY et al (2013) Diffusion tensor tractography of residual fibers in traumatic spinal cord injury: a pilot study. J Neuroradiol 40(3):181-186

18. Shanmuganathan K, Gullapalli RP, Zhuo J et al (2008) Diffusion Tensor MR Imaging in Cervical Spine Trauma. Am J Neuroradiol 29:655-659

19. Cheran S, Shanmuganathan K, Zhuo J et al (2011) Correlation of MR Diffusion Tensor Imaging Parameters with ASIA Motor Scores in Hemorrhagic and Non hemorrhagic Acute Spinal Cord Injury. J Neurotrauma 28(9):1881-1892

20. Kamble RB, Venkataramana NK, Naik AL et al (2011) Diffusion tensor imaging in spinal cord injury. Indian J Radiol Imaging 21:221-224

21. Mohamed FB, Hunter LN, Barakat N et al (2011) Diffusion tensor imaging of the pediatric spinal cord at 1.5T: Preliminary results. Am J Neuroradiol 32: 339-345

22. Hassan H, Maarouf R, Abo-Elela M et al (2020) The role of diffusion tensor imaging (DTI) in spinal cord pathology. Ain Shams Med J 71(1):197-205

23. Li XF, Yang Y, Lin CB et al (2016) Assessment of the diagnostic value of diffusion tensor imaging in patients with spinal cord compression: a metaanalysis. Braz J Med Biol Res 49(1):4769

24. Li XH, Li JB, He XJ et al (2015) Timing of diffusion tensor imaging in the acute spinal cord injury of rats. Sci Rep 5:12639

25. Ellingson BM, Ulmer JL, Kurpad SN et al (2008) Diffusion Tensor MR Imaging in Chronic Spinal Cord Injury. Am J Neuroradiol 29(10):1976-1982

26. Facon D, Ozanne A, Fillard P et al (2005) MR diffusion tensor imaging and fibre tracking in spinal cord compression. Am J Neuroradiol 26:1587-1594

27. Fiani B, Noblett C, Nanney J et al (2020) Diffusion tensor imaging of the spinal cord status post trauma. Surg Neurol Int 11:276

28. Song T, Chen WJ, Yang B et al (2011) Diffusion tensor imaging in the cervical spinal cord. Eur Spine J 20:422-428

\section{Publisher's Note}

Springer Nature remains neutral with regard to jurisdictional claims in published maps and institutional affiliations. 